\title{
Elementos estruturais de um modelo formal dos esportes coletivos de invasão
}

CDD. 20.ed. 796.017

\author{
Leonardo LAMAS* \\ Junior BARRERA** \\ Guilherme OTRANTO** \\ Carlos UGRINOWITSCH* \\ *Escola de Educa- \\ ção Física e Esporte, \\ Universidade de São \\ Paulo. \\ **Instituto de Mate- \\ mática e Estatística, \\ Universidade de São \\ Paulo.
}

\section{Resumo}

0 desenvolvimento de um modelo formal da estratégia e da dinâmica do jogo constitui uma contribuição cientifica original no contexto dos esportes coletivos de invasão. 0 procedimento construtivo de uma estratégia definido no modelo é composto de cinco conjuntos de elementos ordenados em niveis hierárquicos, que suportam o desenho de estratégias capazes de orientar adequadamente uma equipe em um jogo. Uma vez definido o modelo da estratégia, a formalização da dinâmica do jogo permite sua contextualização no momento de sua aplicação para orientar os jogadores no confronto. 0 jogo teve sua estrutura decomposta e suas propriedades dinâmicas fundamentais foram definidas. Dessa forma, a modelagem da dinâmica da oposição e da estratégia se complementam, pois definem os momentos nos quais a informação estratégica é utilizada pelos jogadores. A estrutura formal apresentada inaugura uma linha de pesquisa que poderá contribuir para limitar a subjetividade na definição dos critérios de análise de futuros desenhos experimentais, levando à interpretações e comparações mais acuradas dos resultados dos estudos.

UnITERMOS: Estratégia; Tática; Jogo; Modelagem matemática; Sistemas dinâmicos.

\section{Introdução}

Esportes coletivos de invasão (ECIs) constituem uma classe particular de modalidades esportivas cuja disputa é baseada na oposiçāo entre duas equipes, em um espaço comum, com o objetivo principal de pontuar e impedir o adversário de faze-lo. O sucesso de uma equipe no jogo depende da atuação coletiva de seus jogadores, pois através da sincronização de suas ações é maior a chance de superar o adversário, uma vez que se torna possível realizar ações mais complexas (CANNON-BOWERS \& BOWERS, 2006; WARD \& ECCLES, 2006).

O conjunto de especificações concebidas para dar suporte às açôes coletivas dos jogadores de uma equipe define o conceito de estratégia (GREHAIGNE, Godbout \& Bouthier, 1999; Riera, 1995). A estratégia de uma equipe deve possuir um vasto repertório de opções de comportamentos, que possam gerar incerteza à equipe adversária quanto às suas futuras ações, e produzir assim boas chances de superá-la. Trata-se de um planejamento da ação, que embora sofra modificações em sua implementação como resultado das restrições espaço-temporais mutuamente impostas durante o confronto, possui fundamental influência sobre os padróes de comportamentos observados no jogo.

A execução da estratégia, que obriga os jogadores a promoverem ajustes individuais ao plano estratégico coletivo para atender às necessidades circunstanciais do confronto define o conceito de tática (GREHAIGNE, Godbout \& BOUTHIER, 1999; Riera, 1996). A tática pode ter maior ou menor vinculação à estratégia proposta, consistindo, respectivamente, em adequaçôes dos comportamentos planejados às restrições impostas pelo adversário ou comportamentos dissociados de um plano coletivo. Desta forma, a proficiência de uma equipe para atuar coletivamente parece estar associado tanto às características da estratégia elaborada, que orienta a cooperação entre os jogadores, quanto à eficiência da sua utilização no jogo através da tática.

$\mathrm{Na}$ atualidade, é possível identificar grande número de estudos dedicados à investigação da relação entre os padrôes de comportamentos dos jogadores, por meio da tática, e o sucesso no confronto, em diferentes ECIs 
(Borrie, Jonsson \& Magnusson, 2002; Grehaigne, Bouthier \& David, 1997; Grehaigne, Caty \& Godbout, 2010; Lamas, De Rose Junior, Santana, Rostaiser, Negretti \& Ugrinowitsch, 2011; SAMPaio \& MaÇãs, 2012; Seabra \& Dantas, 2006; Tenga, Holme, Ronglan \& Bahr, 2010a, 2010b; Tenga, Kanstad, Ronglan \& Bahr, 2009). Porém, as diferentes contribuições nesta área, usualmente denominada "análise do jogo", são limitadas pela ausência de formalização dos pressupostos teóricos assumidos nessas investigaçôes. $\mathrm{O}$ fato das análises realizadas, em geral, não descreverem os eventos a partir de um suporte teórico fornecido por uma linguagem formal de representação do fenômeno pode induzir a duas limitações fundamentais: a) imprecisão na descrição dos eventos, pois a ausência de uma estrutura precisa de classificação dos comportamentos observados pode induzir ao aumento do componente subjetivo na avaliação; b) heterogeneidade de critérios definidores de variáveis de análise entre pesquisas, pois as diferenças entre os pressupostos teóricos utilizados pode induzir distintas caracterizações do fenômeno, com diferenças na definição de conceitos e, consequentemente, nas terminologias. Estas deficiências restringem a capacidade dos experimentos conduzidos avaliarem aspectos referentes à globalidade das interações promovidas no confronto entre duas equipes, além de limitarem o avanço no conhecimento pela dificuldade de comparação entre resultados experimentais. A consequência final para a interpretação dos comportamentos do jogo é a possível perda de informaçóes relevantes.

\section{Modelagem da estratégia}

Os principais elementos estruturais de um modelo formal da estratégia desenvolvido por nosso grupo de pesquisa são apresentados a seguir. Para tanto, a estratégia foi definida como um sistema dinâmico de natureza discreta-contínua. Além disso, a modelagem seguiu uma orientação "bottom-up", ou seja, a partir do seu mais detalhado elemento estrutural identificado como relevante, a estrutura formal da estratégia foi elaborada até seu nível mais macro.

\section{Estrutura da ação coordenada dos jogadores}

A ação coletiva de uma equipe foi representada como uma composição de ações individuais, cuja orientação
Em diferentes áreas científicas, a evolução do conhecimento sobre um objeto de estudo é altamente dependente da criação de modelos matemáticos, que formalizem noções empíricas acerca dos fenômenos, e direcionem pesquisas quantitativas sobre o objeto estudado (Duda, HarT \& STORK, 2001).

O efeito desta abordagem metodológica no contexto esportivo pode ser observado pelos resultados decorrentes de estudos inter-disciplinares envolvendo o esporte e áreas como a engenharia, a ciência da computação, entre outras. Os resultados desses estudos podem ser observados no aperfeiçoamento aerodinâmico das bicicletas de corrida (JEUKENDRUP \& MARTIN, 2001) e dos patins utilizados em provas de velocidade no gelo (VAN Ingen Schenau, De Groot, Scheurs, Meester \& De KONING, 1996), bem como na criação de simuladores utilizados no treinamento de pilotos de Fórmula-1 (Wloch \& Bentley, 2004). No caso particular dos esportes coletivos, verifica-se um grande incremento de dados quantitativos disponíveis. As comissões técnicas das equipes tem à disposição imagens de vídeos de jogos e treinamento, medidas do desempenho físico, técnico e tático de cada jogador, etc. Porém, a escassez de elementos formais para a constituição de um modelo teórico desta família de esportes limita o avanço no conhecimento a seu respeito e os potenciais desdobramentos tecnológicos.

A partir de uma perspectiva inter-disciplinar desta natureza, o presente trabalho tem como objetivo comentar alguns elementos estruturais de um modelo sobre os esportes coletivos de invasão, que integra a estratégia e a dinâmica do jogo, conforme proposto por LAMAS (2012).

estratégica, para que seja coordenada com a dos demais jogadores, é explicada pelo conceito de regra de ação. Uma regra de ação é definida como uma declaração condicional com a seguinte estrutura "se <condição $>$, então <ação>”, na qual, a condição é uma interpretação lógica que um jogador faz da percepção do contexto do confronto, enquanto a ação é a seleção de uma ação que será executada com o objetivo de modificar o contexto. Por exemplo, no ataque do futebol, "se $<$ meu companheiro se desmarca em penetração na grande área>, então <eu devo realizar um passe em sua direção>"; em uma ajuda defensiva, "se $<$ meu companheiro de equipe é superado pelo atacante>, então <eu devo recuperar sua posição defensiva>". Dessa forma, as regras de ação formalizam a maneira 
pela qual a especificação estratégica exerce o controle lógico da dinâmica dos jogadores, pois determina a escolha da ação de um jogador, em um dado contexto.

A interação cooperativa entre jogadores de uma equipe pode ser modelada representando o controle da ação de cada jogador por uma lista de regras de ação. Se R1 e R2 são duas regras de ação tais que a condição de R2 é a consequência de R1, então R1 é concatenada com R2. Se R2 é também concatenada com $\mathrm{R} 1$, então $\mathrm{R} 1$ e $\mathrm{R} 2$ são reciprocamente concatenadas. Se R1 é concatenada com R2 e R1 e R2 são regras de ação dos jogadores $\mathrm{p} 1$ e $\mathrm{p} 2$, respectivamente, então p2 é dependente de p1. Se R1 e R2 são reciprocamente concatenados, então p1 e p2 são reciprocamente dependentes. A dependência entre os jogadores é aplicada para definir o conceito de unidade estratégica (UEST), que formaliza a noção de interaçôes cooperativas entre jogadores da equipe. Por exemplo, se exisitir uma dependência recíproca

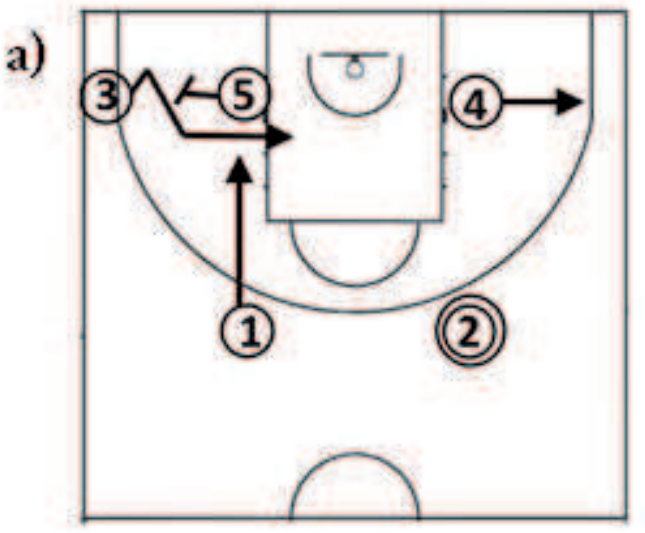

entre os jogadores 1 e 2, então $\{1,2\}$ é uma UEST. A principal propriedade de uma UEST, composta por mais de um jogador, é que todas as açôes do seu conjunto de jogadores são reciprocamente dependentes. Assim, a formação dos grupos é consequência das regras de ação aplicadas.

A FIGURA 1a ilustra as especificações das açōes dos jogadores de uma equipe de basquetebol em uma dada circunstância de jogo. De forma complementar, o diagrama da quadra da FIGURA $1 \mathrm{~b}$, denominado mapa de interaçôes cooperativas, representa as dependências entre jogadores e grupos de jogadores para as especificações de ações da FIGURA 1a. Na FIGURA 1b, há três UESTs: $\{2,3,5\},\{1\}$ e $\{4\}$. Na UEST $\{2$, $3,5\}$, os jogadores têm dependência recíproca, representada pelas setas bi-direcionais que os conectam. As UESTs $\{1\}$ e $\{4\}$ são dependentes da UEST $\{2,3,5\}$, uma vez que 1 e 4 são dependentes de 2, 3 e 5, o que é representado por setas unidirecionais.

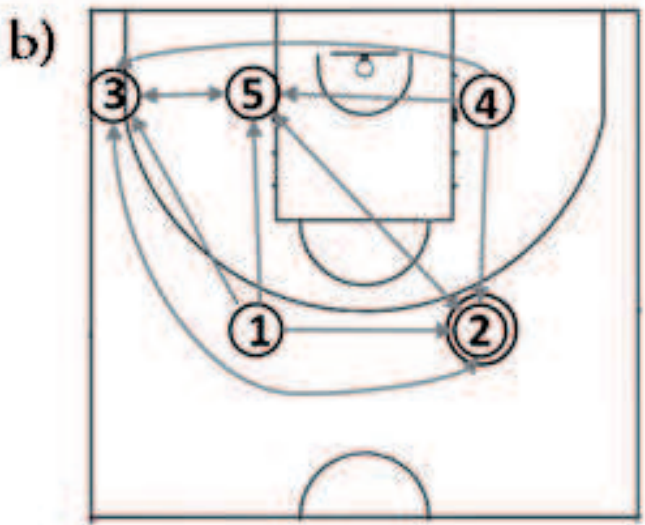

FIGURA 1 - a) descrição das ações dos jogadores de uma equipe; b) descrição das dependências entre jogadores e grupos de jogadores de uma equipe.

Na FIGURA 1a, as ações apresentadas são a consequência da aplicação da regra de ação de cada jogador (e.g., o corta-luz). Na FIGURA 1b, as UESTs apresentadas são especificadas pela concatenação das regras de ação dos jogadores das UESTs, de maneira recíproca (e.g., jogadores 2 , 3 e 5 ) ou unidirecional (e.g., jogador 4 em relação aos jogadores 2, 3 e 5). As relações entre UESTs são especificadas pela concatenação das regras de ação dos jogadores das distintas UESTs (e.g., 1 e 5, 4 e 3).

\section{Definição do estado da estratégia}

A modelagem das sequências de ações dos jogadores em suas respectivas UESTs para cumprir sequências de objetivos estratégicos leva à formalização da estratégia de equipes em ECIs como uma família particular de sistemas dinâmicos discretos-contínuos. De acordo com este formalismo, as especificaçôes estratégicas para os jogadores são contidas em estados do sistema. Um estado é composto por duas estruturas principais: a) estado de movimento; e b) transformação. A formalização destas estruturas será detalhada na sequência.

Um estado de movimento é composto por cinco elementos constituitvos. Primeiro, são especificados todos os jogadores da própria equipe e da equipe adversária no campo de jogo, que possuam relevância semântica ${ }^{1}$ para um dado estado. Segundo, é definida a região de cada jogador no campo de jogo. Cada região representa uma classe de equivalência de posicionamento, na qual qualquer que seja o 
posicionamento de um jogador dentro dessa região tem o mesmo significado semântico. Assim, independente do ponto em que os jogadores se encontrem dentro da suas respectivas regiōes, o estado caracterizado será o mesmo. Terceiro, a dinâmica dos jogadores (i.e., posição tri-dimensional instantânea, velocidade e aceleração em uma dada região). Quarto, o jogador em posse da bola, pois se a equipe tem a posse da bola, ou não, implica diferentes tipos de estratégias (i.e., ofensiva ou defensiva). Quinto, a dinâmica da bola definida por sua posição instantânea, velocidade e aceleração. Dois estados de movimento são diferentes quando ao menos um de seus elementos constitutivos diferir.

As FIGURAS $2 \mathrm{a}$ e $2 \mathrm{c}$ apresentam estados de movimento típicos. $\mathrm{Na} 2$ a e 2c, o segundo círculo em torno do jogador 2 representa a bola. As elipses externas a todos os jogadores em 2a e $2 \mathrm{c}$ indicam as regiōes de posicionamento dos jogadores (i.e., classe de equivalência de posicionamento). Jogadores defensivos são representados por círculos com traços pretos adjacentes, simbolizando seus braços.

A transformação especifica as tarefas dos jogadores da equipe (i.e., trajetória no campo de jogo e respectivas habilidades esportivas realizadas) e suas dinâmicas. Há transformaçōes nas quais nenhum jogador se desloca, mas ocorre uma modificação na semântica da equipe. Por exemplo, se a bola é passada, há uma mudança na ação de dois jogadores e, possivelmente, em suas dinâmicas (FIGURA 2b1). Ainda, a transformação especifica as interações cooperativas entre alguns dos jogadores, que constituem as UESTs da transformação. A coordenação das UESTs define a organização coletiva da equipe, cujas especificaçôes são modificadas na transformação seguinte. As UESTs são especificadas por diagramas de setas, com notação idêntica à da FIGURA 1. A especificação completa de uma transformação é ilustrada nas FIGURAS 2b1 e 2b2.

Um estado é uma ponte que recebe como entrada um estado de movimento, o estado de movimentoentrada, modifica essa entrada pela transformação, que reposiciona e atualiza a dinâmica dos jogadores da equipe, definindo a constituição do estado de movimento subsequente. No novo estado de movimento, o estado de movimento-saída, são definidas as regiōes dos jogadores, suas dinâmicas e a posse e dinâmica da bola. A dinâmica dos jogadores é definida pela dinâmica final da transformação. Além disso, suas regiōes são planejadas de acordo com a posição final da transformação. a) estado de movimento-entrada;

b) transformação: b1) deslocamentos; b2) interações cooperativas; c) estado de movimento-saída.

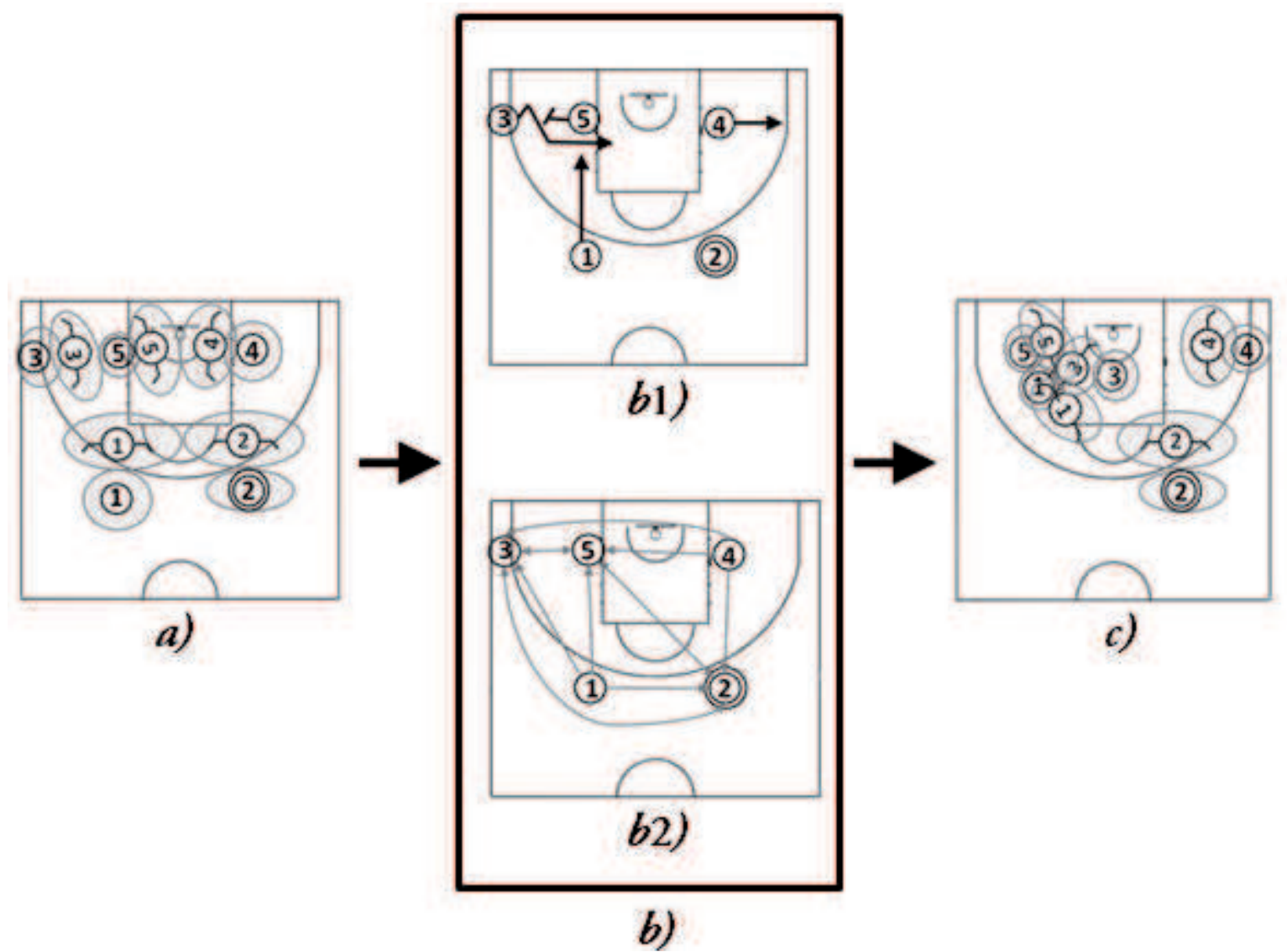

FIGURA 2 - Decomposição de um estado em seus elementos constituitvos, ilustrado por diagramas convencionais de quadras de basquetebol. 
De acordo com a definição de estado, deve haver um único mapa de interações cooperativas para cada transformação, pois uma única regra de ação pode ser aplicada por cada jogador em um estado no nível do planejamento estratégico das ações. Se uma movimentação planejada requer uma concatenação complexa entre os jogadores, sua especificação estratégica deve ser descrita ao longo de uma sequência de estados, na qual cada estado contém apenas um mapa de interaçôes cooperativas. Para se desenhar uma sequência consistente de estados, é necessário que dois estados subsequentes sejam compatíveis, ou seja, o estado de movimento-saída do primeiro seja idêntico ao estado de movimento-entrada do segundo. Uma jogada é modelada por uma sequência tal que cada par de estados subsequentes é compatível. No jogo, as restrições impostas pelo confronto podem levar à execução de regras de ação distintas das planejadas e gerar estados diferentes daqueles especificados na estratégia.

\section{Representação dos estados da estratégia}

As estratégias desenhadas segundo este formalismo são compostas por um conjunto finito de estados e conexôes entre pares de estados compatíveis e podem ser representadas por um grafo ${ }^{2}$. Cada nó do grafo de uma estratégia é associado a um estado e os arcos do grafo conectam nós associados a estados adjacentes. Um estado armazena a semântica de um fragmento de uma jogada e o grafo representa as conexões entre fragmentos para definir uma jogada completa. A representação pelo grafo constitui uma alternativa útil para a comissão técnica identificar a estrutura geral da estratégia planejada e interpretar cada uma das sequências de estados à luz da estratégia completa.

Para ilustrar esse conceito, na sequência, uma variação da conhecida estratégia ofensiva no basquetebol, denominada ofensiva em triângulos (WINTER, 1962), será apresentada por um grafo (FIGURA 3). Uma região particular do grafo, delimitada com uma linha pontilhada, indica dois caminhos distintos (destacados abaixo do grafo), cujos conteúdos serão especificados na sequência. No início da FIGURA 3 pode ser observado que os dois caminhos têm um início comum no primeiro nó (i.e., que contém a especificação do estado E1). No segundo nó (E2), dentre os sete caminhos possíveis, são destacados os dois que terminam, respectivamente em E5 e E8.

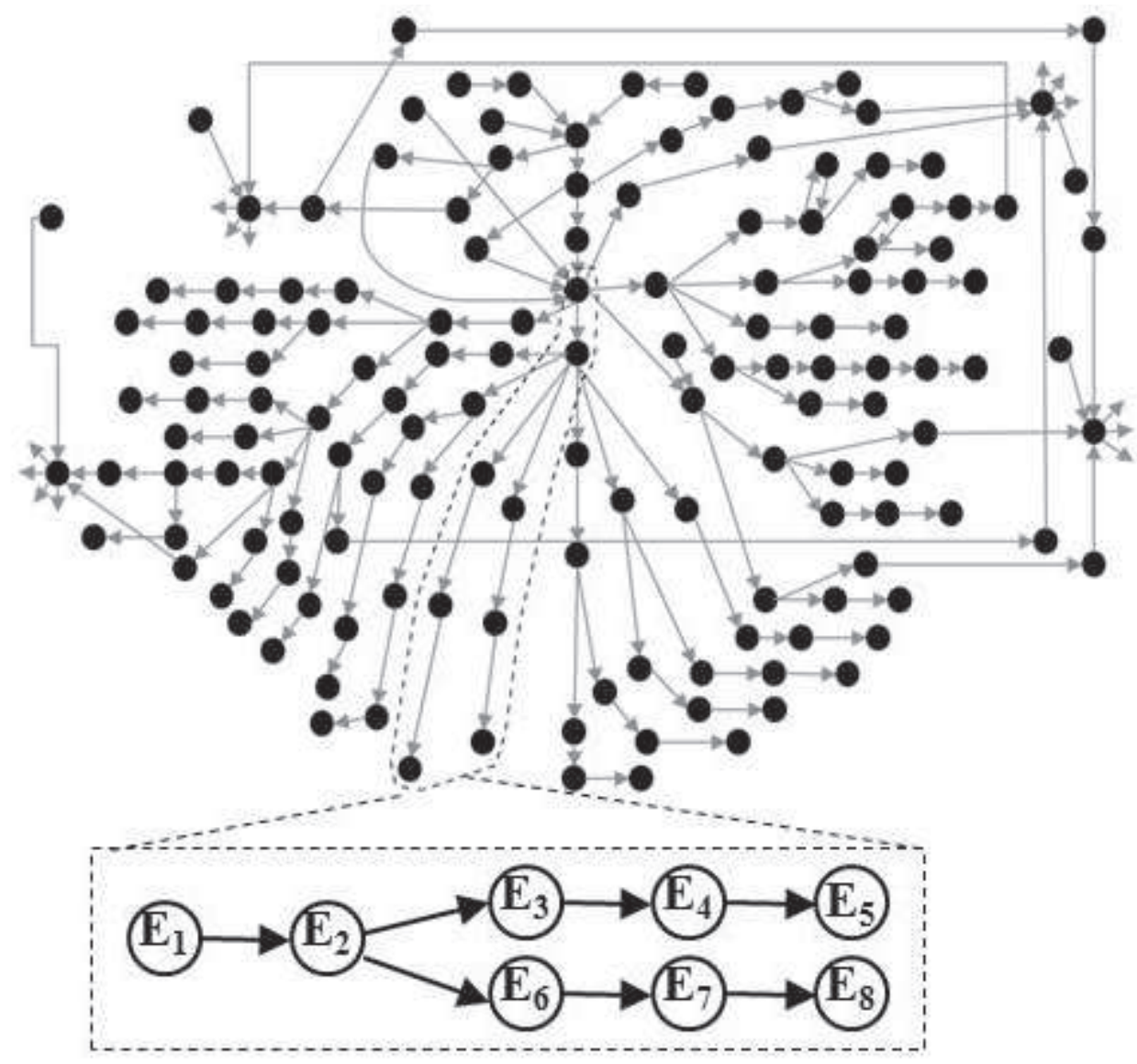

FIGURA 3 - Uma variação da estratégia ofensiva do basquetebol, a ofensiva em triângulos, representada por um grafo, no qual: $\mathrm{E}_{\mathrm{n}}$ designa o nome de um estado em um caminho do grafo. 
Círculos com números no interior: jogadores; setas pretas: deslocamentos;

Círculos cinzas: regiões de cada jogador;

Segundo círculo externo: jogador da bola.
As sequências destacadas na região do grafo selecionada na FIGURA 3 são especificadas na FIGURA 4. Para este propósito, foi utilizada uma notação condensada para o estado. Cada sequência de dois diagramas de quadras de basquetebol corresponde a um estado no qual o estado de movimento-entrada e a transformação estão no primeiro diagrama e o estado de movimento-saída é o estado de movimento-entrada do diagrama subsequente. No primeiro diagrama de quadra da FIGURA 4 (Q1) é planejado que o jogador 3 deva fazer um passe (seta negra pontilhada no diagrama) para o jogador 5 (números dos jogadores em itálico).
Um círculo extra ao redor do número de cada jogador indica a posse da bola. $\mathrm{O}$ passe interior para 5 é o evento que dispara o deslocamento de 1,2,3 e 4 (seta negra contínua) representado em Q2. Em Q2, 1 e 3 realizam cortes subsequentes sobre 5 , enquanto 2 desloca-se para o lado oposto à bola e 4 aproxima-se do garrafão. Após, em Q3, 5 permanece com a bola, 1 realiza bloqueio para 4,4 desmarca-se através do bloqueio de 1, 2 desloca-se para criação de linha de passe do lado da bola e 3 termina seu corte no lado oposto do garrafão. Nos demais diagramas, é empregada a mesma notação para descrever os eventos especificados.

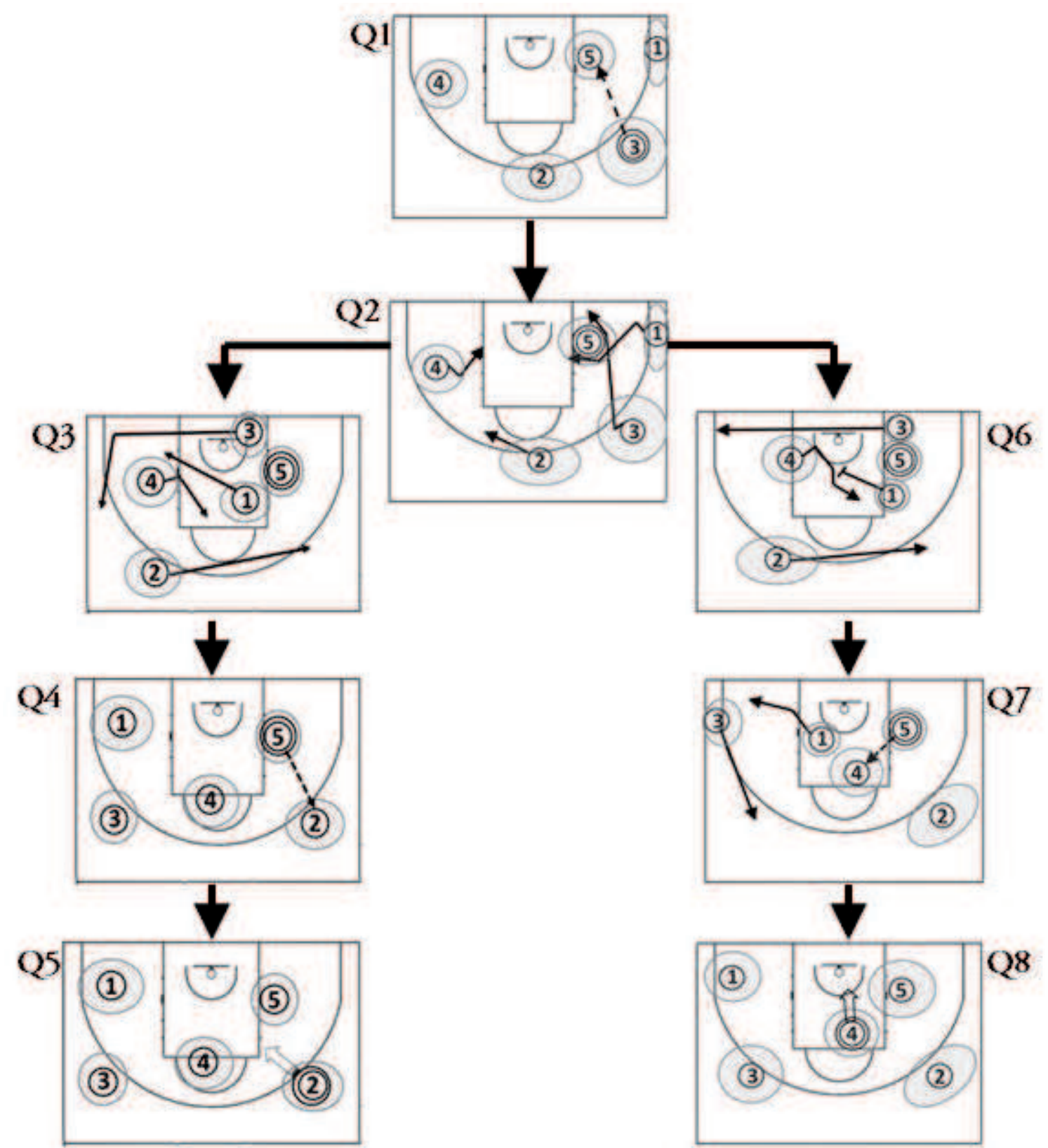

FIGURA 4 - Representação de duas sequências de estados de uma variação da estratégia ofensiva de basquetebol denominada ofensiva em triângulos. 
Um elemento estrutural do modelo proposto é a adição de dois tipos de delimitadores ${ }^{3}$ para restrição da família de sistemas dinâmicos que correspondem às estratégias adequadas para os ECIs. Com a aplicação destes delimitadores é possível avaliar estratégias e aquelas que atendem somente aos elementos estruturais anteriormente mencionados são insuficientes para orientar de forma eficiente uma equipe. Os dois delimitadores estabelecidos no modelo são: a) as fases do jogo (e.g., ataque, defesa, disputa), assim como suas respectivas decomposições (e.g., o contra-ataque em relação ao ataque, a transição defensiva em relação à defesa). É necessário que a estratégia contemple especificaçôes para todas as fases do jogo, preferencialmente, com semelhante grau de complexidade entre elas. $\mathrm{Ou}$ seja, a equipe não deve ser, por exemplo, mais forte para atacar do que para defender, do ponto de vista da estratégia planejada; b) os tipos de estrutura de conexões entre os nós da representação pelo grafo. Há necessidade de diversificação dos caminhos no grafo que conduzam às situaçôes de finalização. Isto significa que vários estados no grafo devem conectarse a mais de um estado, de maneira a aumentar a imprevisibilidade para o adversário quanto à ação futura a ser realizada.

\section{Modelagem da dinâmica do jogo}

A estrutura apresentada contextualiza a estratégia como a base de dados acessada para subsidiar as decisões dos jogadores e permitir que eles atuem coletivamente, durante as diferentes circunstâncias do confronto. A influência da estratégia sobre a dinâmica do jogo é representada na estrutura do jogo descrita a seguir.

\section{Linguagem formal para modelagem da dinâmica do jogo}

A dinâmica do jogo foi modelada pela sua decomposição no planejamento da equipe e no confronto, quando os planejamentos são efetivamente executados. Para realizar a decomposição foi utilizado uma linguagem formal denominada notação para modelagem de processos de negócios (NMPN), que possui um conjunto genérico de conceitos e possibilidades de modelagem. $\mathrm{O}$ uso do formalismo da linguagem será exemplificado através de um fluxo que apresenta o processo competitivo em esportes coletivos, considerando o jogo, sua relação com a estratégia e outros elementos relevantes (FIGURA 5).

Conforme ilustrado na FIGURA 5, objetos de $f l u x o$, tais como processos e portas representam os principais elementos lógicos utilizados para definir o comportamento do fluxo. Um processo representa uma unidade de trabalho, que pode, ou não, ser decomposta em outros processos (e.g., o processo "Jogo" é decomposto na FIGURA 5). Portas podem ser de dois tipos: exclusivas e paralelas. Uma porta exclusiva é um local do processo no qual o fluxo pode tomar um de dois caminhos alternativos. Uma porta paralela é aplicada para sincronizar duas sequências de fluxo paralelas para um fluxo de saída comum. Um artefato fornece informação adicional sobre a execução de um processo. Um dado é o principal tipo de artefato, sendo utilizado para representar como uma certa informação é gerada e consumida por diferentes processos. A conexão entre um dado e um processo é descrita com um objeto de conexão. Objetos de conexão são de dois tipos: sinais de controle e associaçôes, representadas por setas contínuas e pontilhadas, respectivamente. Sinais de controle conectam um processo a outro, indicando a ordem em que serão executados, enquanto associações conectam um processo a um artefato, indicando o processo do qual o dado foi originado. Todo processo tem seu início indicado por um círculo branco e seu fim indicado por um círculo cinza escuro, dos quais, respectivamente, parte e chega o sinal de controle. O término de um processo indica o final da decomposição dos conteúdos em dado nível. Os elementos gráficos que representam os conceitos descritos são indicados na legenda da FIGURA 6. O "software" utilizado para aplicação da linguagem é denominado BizAgi ${ }^{\circledR}$ (Buckinghamshire, UK). 
Retângulos são processos;

Losangos são testes Booleanos;

Estruturas em formato de papel são dados;

Setas contínuas são sinais de controle;

Setas pontilhadas são associações.

EST: estratégia;

ESTjogo: estratégia especificada para um jogo.

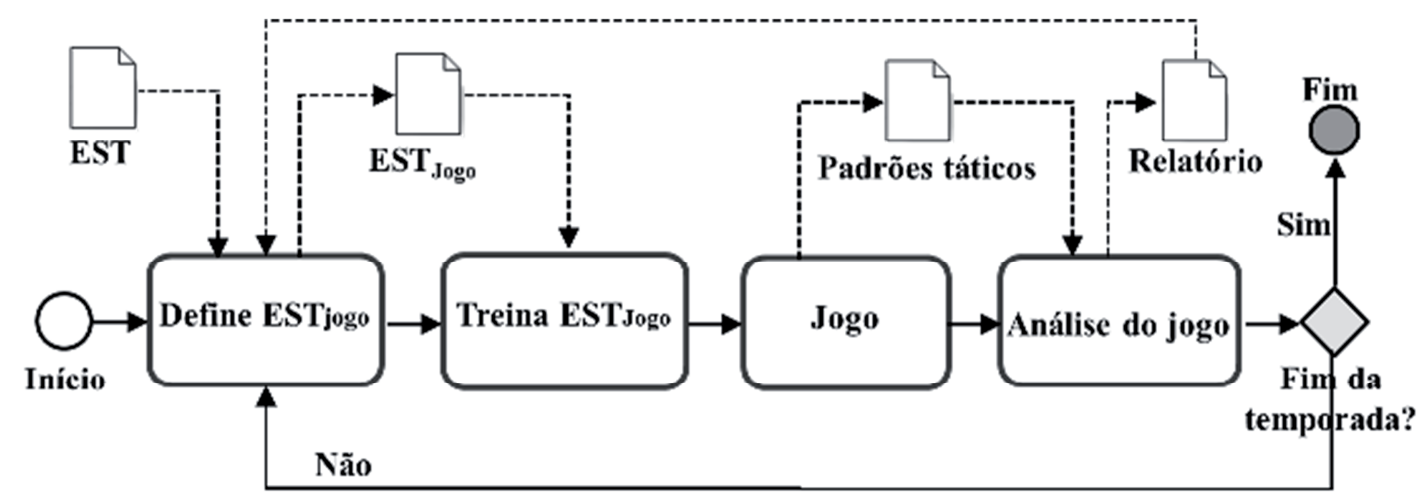

FIGURA 5 - Processo competitivo em esportes coletivos de invasão, com seus fluxos de controle e de dados.

\section{Estrutura dinâmica do jogo}

No modelo da dinâmica do jogo (FIGURA 6), a estratégia concebida pela comissão técnica define a estrutura do comportamento inteligente coletivo de cada equipe e seus objetivos, sendo em cada momento do jogo interpretada e aplicada pelos jogadores. $\mathrm{Na}$ aplicação da estratégia, em certas circunstâncias os jogadores podem não ser aptos a responder adequadamente às exigências da oposição, o que pode levar à diminuição da organização da equipe. A estrutura dos principais elementos da organização da equipe e das restrições impostas pela oposição, que conjuntamente caracterizam a dinâmica do jogo, serão apresentadas na sequência.

A representação da dinâmica do jogo é iniciada com o "Posicionamento inicial no campo de jogo" (FIGURA 6). Este processo tem como entrada os dados referentes ao sub-conjunto da estratégia completa (dado-SC-ESTjogo) de cada uma das equipes (A e B), que as respectivas comissóes técnicas decidem utilizar em um jogo específico. Em um exemplo, supondo que para enfrentar certa equipe, identifica-se que defesa por zona não é uma alternativa adequada, então no dado-SC-ESTjogo selecionado para esta partida, as sequências de estados da estratégia da equipe correspondentes à defesa por zona não serão incluidas. Com isso, no âmbito defensivo, o sub-conjunto da estratégia será constituído por outras defesas que não por zona. $\mathrm{O}$ "Posicionamento inicial no campo de jogo" recebe como entrada também o dado- $\mathrm{J}_{\mathrm{i}-1}$ (i > 0), referente à semântica da última intervenção do árbitro (e.g., marcação de uma falta, violação ou uma bola que saiu pela lateral após um passe errado), que influi diretamente no tipo de posicionamento para o reinício (e.g., no futebol, é bastante diferente o posicionamento para reinício com um escanteio ou com tiro de meta). Com estes dados de entrada, é determinado o posicionamento no campo de jogo a ser empregado para o próximo intervalo da oposição. Note que a representação do jogo aqui realizada corresponde a cada intervalo entre interrupções. Assim, o primeiro intervalo representável é o período transcorrido entre o início do jogo e a primeira interrupção pelo árbitro. $\mathrm{O}$ índice $\mathrm{i}>0$ do dado- $\mathrm{J}_{\mathrm{i}-1}$ implica valor zero para o dado- $\mathrm{J}_{\mathrm{i}-1}$ na primeira iterada do fluxo, pois trata-se do início do jogo, ou reinício de um dos seus períodos, e não há informação a ser transmitida. Nas iteradas subsequentes do fluxo, o dado terá algum valor semântico, pois a interrupção que leva à necessidade de um novo posicionamento para o re-início terá ocorrido como consequência de algum tipo de intervenção do árbitro. A saída do processo é o dado-Si que especifica os parâmetros do estado caracterizado (i.e., região ocupada por cada jogador no campo de jogo, dinâmica dos jogadores e da bola, e posse da bola).

O processo seguinte representa a definição do plano de cooperação entre os jogadores da equipe. Quando "Jogadores planejam cooperação" ocorre imediatamente após o início do jogo, o processo especifica um plano apenas para os primeiros instantes da oposição, até que a posse de bola e a configuração de ambas equipes no campo de jogo seja estabelecida. Nos re-inícios seguintes, este processo definirá planos de ação podendo envolver sequências mais ou menos longas de estados a serem executadas durante a oposição. Para tanto, "Jogadores planejam oposição" utiliza o dado- $S_{i-1}(i>0)$, sendo que o valor zero da primeira iterada corresponde à informação do posicionamento inicial, que antecede o reinício da oposição. Como saída, o processo que efetua o planejamento da cooperação devolve as regras de ação que cada jogador deverá seguir para execução do próximo estado da sequência planejada.

O fluxo é então sincronizado e entra no processo "Oposição", indicando o enfrentamento entre as equipes. $\mathrm{O}$ dado regras-de-ação das duas equipes é a entrada da "Oposição", indicando o conjunto de 
declarações lógicas do tipo: se <condição>, então $<$ ação >, especificadas para cada jogador, que orientarão seus comportamentos nos instantes seguintes. O dado$\mathrm{S}_{\mathrm{i}-1}$ também é entrada deste processo, pois a oposição entre as equipes ocorre a partir do contexto atual, descrito pelos parâmetros do estado presente. A saída da "Oposição" é o dado-Si, que representa o estado produzido pelo jogo, após a interação das equipes.

$\mathrm{Na}$ sequência, o processo "Árbitro verifica as açôes" recebe como entrada $S_{i}$ e indica o momento em que o resultado das interações na oposição é analisada. Em caso de necessidade, o árbitro pode interceder e interromper o jogo, conforme indicado pela sequência do fluxo, ao passar pelo teste Booleano "Interrupção?". Caso não haja interrupção, o fluxo retorna para "Jogadores planejam cooperação", onde o estado seguinte será especificado por meio da definição das regras de ação de cada jogador. Em circunstâncias nas quais o árbitro interrompe o jogo, o tipo de interrupção é comunicada, conforme indica a sequência do fluxo para "Árbitro descreve tipo de interrupção". Este processo gera o dado- $\mathrm{J}_{\mathrm{i}}$, que será novamente utilizado no "Posicionamento incial no campo de jogo”. A dinâmica exposta no fluxo representado pode ser decomposta levando em conta os principais processos que definem o jogo.

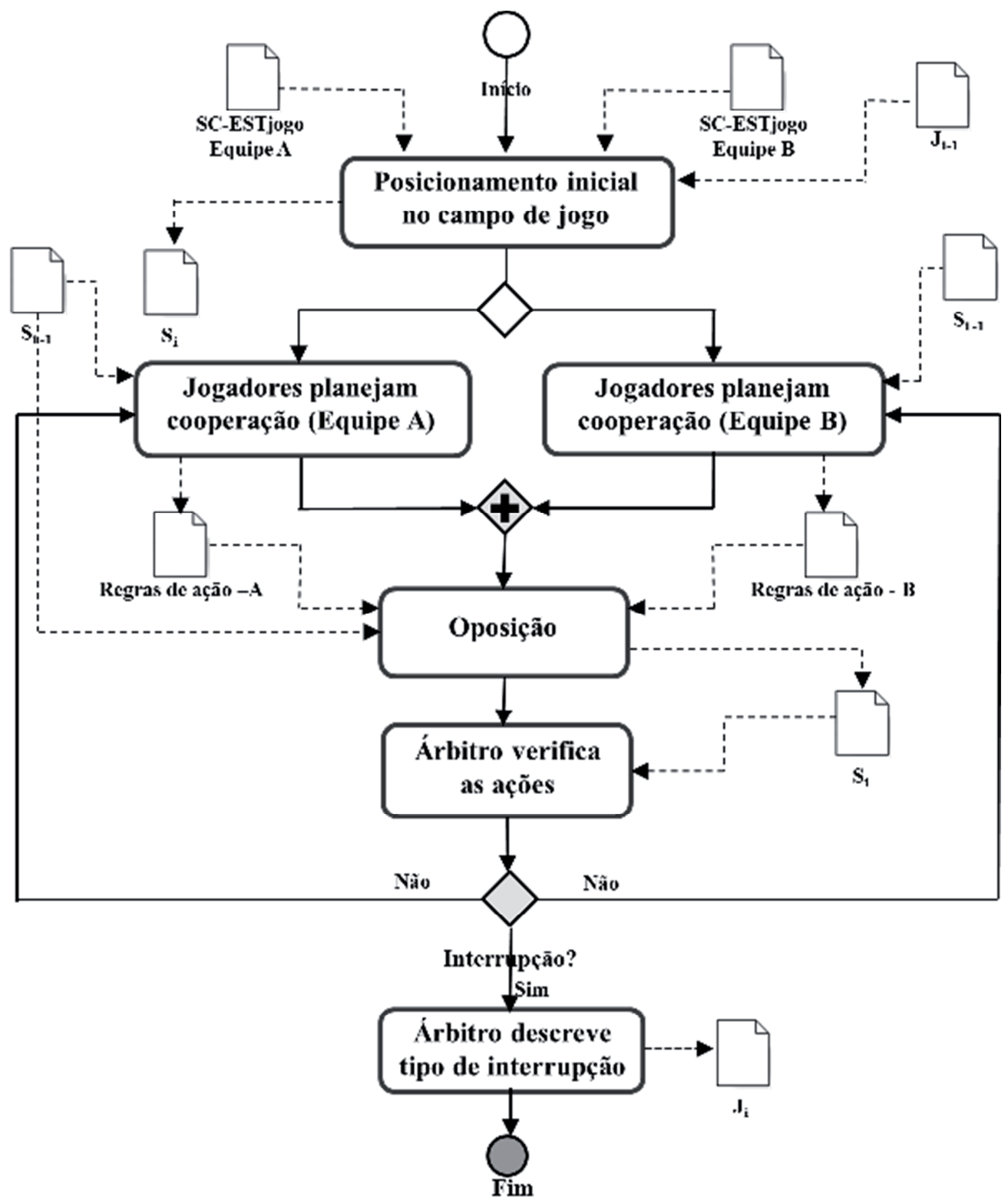

SC-ESTjogo: Sub-conjunto da estratégia para o jogo;

$\mathrm{S}_{\mathrm{i}-1}$ e $\mathrm{S}_{\mathrm{i}}$ : dados referentes aos parâmetros do estado do jogo atual e do estado gerado após a oposição, respectivamente;

$\mathrm{J}_{\mathrm{i}-1}$ e J: dados referentes à semântica da intervenção do árbitro no momento atual e após a oposição, respectivamente.

FIGURA 6 -Dinâmica do jogo: fluxos de controle e de dados. 


\section{Discussão}

O desenvolvimento de um modelo formal da estratégia e da dinâmica do jogo constitui uma contribuição científica original no contexto dos esportes coletivos de invasão. Até então, o desenho da estratégia de uma equipe dependia principalmente da experiência da comissão técnica, por ausência de um suporte teórico estabelecido para orientar esta elaboração. Da mesma forma, em diversos estudos, e também na prática da análise de jogo, apenas aspectos específicos eram considerados nas análises. Assim, a formalização da estratégia e da dinâmica do jogo definem uma parte importante da estrutura do processo competitivo, que poderá fornecer suporte teórico a pesquisas que objetivem determinar as razôes do sucesso no confronto.

$\mathrm{Na}$ modelagem da estratégia um elemento lógico fundamental é a regra de ação, que descreve as decisões de um jogador. As regras de ação também são usadas para a especificação da transformação de um estado e, consequentemente, da sequência de transformações de estados em uma estratégia. A modelagem realizada é suficiente para explicar a aplicação dinâmica das regras de ação durante uma situação do jogo, o que constitui um avanço em relação à noção de uma estrutura lógica que orienta o comportamento dos jogadores descrita em trabalhos prévios (Grehaigne \& Godbout, 1995; Lebed, 2007; MCPherson, 1999a, 1999b; ). A concatenação das regras de ação permite também formalizar a noção de grupos cooperativos através do conceito de UESTs e suas interações. A evolução dos grupos cooperativos caracteriza uma decomposição dinâmica da estratégia, com importante impacto no seu desenho.

A formalização do conceito de UEST e sua orquestração com as demais UESTs, a cada estado da estratégia, contribui para o avanço do entendimento teórico sobre a constituição e dinâmica de grupos funcionais, cujos parâmetros vêm sendo sistematicamente investigados tanto no contexto esportivo (Passos, Araujo, Davids, Gouveia, Milho \& Serpa, 2008; Passos, Milho, Fonseca, Borges, Araujo \& DaVIDS, 2011) quanto militar (Fiore \& Salas, 2006; Pedersen \& COOKE, 2006) mas carecendo de uma formulação teórica mais abrangente que contextualize o conceito frente à estratégia e à tática no confronto.

O desenho das estratégias embasado pelo formalismo proposto permite sua representação por grafos, nos quais o formalismo dos estados é conservado. Os conceitos de estado e grafo definem a estrutura para o desenho de estratégias. Entretanto, esta estrutura pode permitir a especificação de uma família muito mais ampla de estratégias do que aquelas que representam estratégias eficientes. Por isso, um conjunto de delimitadores são requisitos para o desenho de uma estratégia eficiente. A família resultante de estratégias possui algumas características necessárias: completude estratégica para as fases do jogo e estruturas topológicas particulares do grafo (i.e., números de arestas de entrada e saída dos diferentes tipos de nós).

Os elementos formais da modelagem apresentados caracterizam uma metodologia hierárquica para representar construtivamente uma estratégia. Um conjunto de ações é representado pela concatenação de movimentos (i.e., produzido por regras de concatenação). Um conjunto de UESTs é representado pela concatenação de ações. Um conjunto de transformações é representado pela concatenação de UESTs. A concatenação de um sub-conjunto de estados compatíveis cria uma movimentação bem especificada. Assim, este procedimento construtivo é composto de cinco conjuntos de elementos ordenados em níveis hierárquicos: a) habilidades de movimentos básicos; b) habilidades esportivas; c) concatenação das regras de ação dos jogadores nas UESTs; d) concatenação de UESTs na transformação do estado; e) concatenação de estados. Deste modo, esta estrutura construtiva suporta o desenho de estratégias capazes de orientar adequadamente uma equipe em um jogo.

Uma vez definido o modelo da estratégia, a formalização da dinâmica do jogo permite sua contextualização no momento de sua aplicação para orientar os jogadores no confronto. $\mathrm{O}$ jogo teve sua estrutura decomposta e suas propriedades dinâmicas fundamentais foram definidas. Estudos que abordaram aspectos do fenômeno anteriormente, investigaram, de forma intuitiva, algumas noções de controle do planejamento das ações (LEBED, 2007), ou ainda formalizaram alguns aspectos de sua dinâmica (Perse, Kristan, Kovacic, Vuckovic \& Pers, 2009). No entanto, a abrangência da representação do jogo possui aspectos originais tanto na abordagem de modelagem utilizada quanto no seu nível de detalhamento.

A utilização da estratégia é feita pelos jogadores a cada mudança de fase do jogo (i.e., ataque, defesa, disputa) pois nesses momentos é feita uma escolha quanto à movimentação a ser utilizada, a partir das opçôes disponíveis na estratégia (SC-ESTjogo) e das circunstâncias do confronto. Ao término de uma 
movimentação, nova decisão deve ser tomada para que outra sequência de estados seja escolhida e a correpondente movimentação realizada. Para realizar a escolha da sequência subsequente os jogadores acessam: a) os dados do SC-ESTjogo; b) dado do estado real do jogo produzido na oposição (i.e., dinâmicas dos jogadores); c) metadado contendo demais informaçóes como o aproveitamento da equipe em determinada movimentação, o tempo restante de jogo, a diferença no placar, etc.

Após o término do planejamento, a interação dos jogadores de ambas equipes é modelado como um sistema dinâmico contínuo. Cada jogador executa sua respectiva regra de ação planejada, sendo o resultado da interação com o adversário a geração de um novo estado do jogo (i.e., Si-real). Os estados são produzidos constantemente e comparados com os estados existentes no SC-ESTjogo, nos momentos de consulta da estratégia (indicado pelo sinal de controle na FIGURA 6). Quando não há diferenças significativas entre o estado do jogo e os estados da SC-ESTjogo, um novo estado do SC-ESTjogo é selecionado. Por outro lado, quando há diferenças, uma nova movimentação deve ser selecionada.

No modelo proposto, a dinâmica do jogo contempla distintas instâncias de decisão, cuja estrutura hierárquica foi modelada. No mais alto nível de generalização, a comissão técnica define o SC-ESTjogo, ou seja, as especificaçôes estratégicas a serem empregadas no jogo. O SC-ESTjogo é a entrada de dados que orienta as açôes de cada equipe durante o confronto. Sua representação segue o formalismo do modelo da estratégia e, portanto, contempla seus delimitadores. Dessa forma, contém especificaçōes para todas as fases do confronto, assim como possui diferentes tipos de estados quanto ao número de entradas e saídas de cada um deles, aumentando a diversidade de possibilidades de açôes dos jogadores. Para cada intervalo entre duas interrupções subsequentes do jogo a comissão técnica pode selecionar um novo sub-sistema do SC-ESTjogo. Portanto, a modelagem da dinâmica da oposição e da estratégia se complementam, pois definem os momentos nos quais a informação estratégica é utilizada pelos jogadores. Em uma perspectiva genérica para todos os ECIs, indicam a relação entre planejamento e execução das açôes, constituindo uma contribuição inédita para a teoria desta família de esportes.

Dessa forma, a estrutura formal apresentada inicia uma linha de pesquisa que poderá contribuir para o embasamento de estudos quantitativos com diferentes escopos na temática dos esportes coletivos, limitando a subjetividade na definição dos desenhos experimentais e critérios de análise e, consequentemente, na interpretação e comparação de resultados.

\section{Notas}

1. No contexto deste trabalho, o termo semântica denota o significado estratégico ou tático do elemento formal discutido.

2. Um grafo é definido pelo par ordenado $G=(N, A)$ que contempla um conjunto $N$ de nós e um conjunto $A, A \subset N$ x $N$, de arcos.

3. Um delimitador de um sistema dinâmico é uma condição requerida para satisfazer a solução de um problema, no caso, a definição de estratégias adequadas para orientar uma equipe.

\section{Abstract}

\section{Structural elements of a formal model for invasion team sports}

The development of a formal model integrating the strategy and the match dynamics constitute an original scientific contribution in the context of invasion team sports. The constructive procedure of a team strategy defined by the model is composed of sets of hierarchically organized elements, which support the design of strategies that can adequatelly orient a team in a match. Once defined the strategy model, the formalization of the match dynamics contextualizes the strategy on its application to orient players in the opposition. The match structure was decomposed and its main dynamical properties were defined. Thus, the modeling of the match dynamics complements the strategy model, since the first of them defines the circunstances in which the strategic information is applied by the players. The formal model presented inaugurates a 
research field which may contribute to limit the subjectivity in the definition of analysis criteria of future research designs, leading to more accurate interpretation and comparisons between results of studies.

UnITERMs: Strategy; Tactics; Match; Mathematical modeling; Dynamic systems.

\section{Resumen}

Elementos estructurales de un modelo formal del los deportes de equipo y invasion

El desarrollo de un modelo formal de la estrategia y de la dinámica del juego es una contribución científica original en el contexto de los deportes de equipo e invasión. El procedimiento constructivo de una estrategia definida en el modelo se constituye de cinco conjuntos de elementos dispuestos en niveles jerárquicos, que soportan al diseño de estrategias para orientar correctamente un equipo en un juego. Una vez definido el modelo de la estrategia, la formalización de la dinámica del juego permite su contextualización en el momento de su aplicación para orientar a los jugadores en el enfrentamiento. El juego tuvo su estructura descompuesta y sus propiedades dinámicas fueron definidas. Por lo tanto, el modelo de la estrategia y la dinámica de la oposición definen los momentos en que se utiliza información estratégica por los jugadores. La estructura formal presentada comienza una línea de investigación que puede ayudar a reducir la subjetividad en la definición de los criterios para el análisis de los futuros diseños experimentales, dando lugar a interpretaciones más precisas y comparaciones mas acuradas de los resultados del los estudios.

Palabras Clave: Estrategia; Tatica; Juego; Modelación matemática; Sistemas dinámicos.

\section{Referências}

BORRIE, A.; JONSSON, G.K.; MAGNUSSON, M.S. Temporal pattern analysis and its applicability in sport: an explanation and exemplar data. Journal of Sports Sciences, London, v.20, n.10, p.845-52, 2002.

CANNON-BOWERS, J.A.; BOWERS, C. Applying work team results to sports teams: opportunities and cautions. International Journal of Sport and Exercise Psychology, Abingdon, v.4, p.363-69, 2006.

DUDA, R.; HART, P.; STORK, D. Pattern classification. New York: John Wiley and Sons, 2001.

FIORE, S.M.; SALAS, E. Team cognition and expert teams: developing insights from cross-disciplinary analysis of exceptional teams. International Journal of Sport and Exercise Psychology, Abingdon, v.4, p.369-75, 2006.

GREHAIGNE, J.F.; BOUTHIER, D.; DAVID, B. Dynamic-system analysis of opponent relationships in collective actions in soccer. Journal of Sports Sciences, London, v.15, n.2, p.137-49, 1997.

GREHAIGNE, J.F.; CATY, D.G.; GODBOUT, P. Modelling ball circulation in invasion team sports: a way to promote learning games through understanding. Physical Education and Sport Pedagogy, Abingdon, v.15, n.3, p.257-70, 2010. GREHAIGNE, J.F.; GODBOUT, P. Tactical knowledge in team sports from a constructivist and cognitivist perspective. Quest, New York, v.47, p.490-505, 1995.

GREHAIGNE, J.F.; GODBOUT, P.; BOUTHIER, D. The foundations of tactics and strategy in team sports. Journal of Teaching in Physical Education, Champaign, v.18, p.159-74, 1999.

JEUKENDRUP, A.E.; MARTIN, J. Improving cycling performance: how should we spend our time and money. Sports Medicine, Auckland, v.31, n.7, p.559-69, 2001.

LAMAS, L. Modelagem estratégico-tática em esportes coletivos de invasão: aplicação ao basquetebol. 2012. Tese (Doutorado) - Escola de Educação Física e Esporte, Universidade de São Paulo, São Paulo, 2012.

LAMAS, L.; DE ROSE JUNIOR, D.; SANTANA, F.; ROSTAISER, E.; NEGRETTI, L.; UGRINOWITSCH, C. Space creation dynamics in basketball offence: validation and evaluation of elite teams. International Journal of Performance Analysis in Sports, Cardiff, v.11, p.71-84, 2011.

LEBED, F. A dolphin only looks like a fish: players' behavior analysis is not enough for game understanding in the light of systems approach - a response to the reply by McGarry and Franks. European Journal of Sport Science, Abingdon, v.7, n.1, p.55-62, 2007. 
McPHERSON, S. Expert-novice differences in performance skills and problem representations of youth and adults during tennis competition. Research Quarterly for Exercise and Sport, Washington, v.70, n.3, p.233-63, 1999a.

. Tactical differences in problem representations and solutions in collegiate varsity and beginner female tennis players. Research Quarterly for Exercise and Sport, Washington, v.70, n.4, p.369-84, 1999 b.

PASSOS, P.; ARAUJO, D.; DAVIDS, K.; GOUVEIA, L.; MILHO, J.; SERPA, S. Information-governing dynamics of attacker-defender interactions in youth rugby union. Journal of Sports Sciences, London, v.26, n.13, p.1421-29, 2008. PASSOS, P.; MILHO, J.; FONSECA, S.; BORGES, J.; ARAUJO, D.; DAVIDS, K. Interpersonal distance regulates functional grouping tendencies of agents in team sports. Journal of Motor Behavior, Washington, v.43, n.2, p.155-63, 2011. PEDERSEN, H.K.; COOKE, N.J. From battle plans to football plays: Extending military team cognition to football International Journal of Sport and Exercise Psychology, Abingdon, v.4, p.422-46, 2006.

PERSE, M.; KRISTAN, M.; KOVACIC, S.; VUCKOVIC, G.; PERS, J. A trajectory-based analysis of coordinated team activity in a basketball game. Computer Vision and Image Understanding, Orlando, v.113, n.5, p.612-21, 2009.

RIERA, J.R. Análises de la táctica deportiva. Apunts: Educación Fisica y Deportes, Barcelona, v.40, p.47-60, 1995. . Estrategia, táctica y técnica deportivas. Apunts: Educación Fisica y Deportes, Barcelona, v.39, p.45-56, 1996.

SAMPAIO, J.; MAÇÃS, V. Measuring tactical behaviour in football. International Journal of Sports Medicine, Stuttgart, v.33, p.395-401, 2012.

SEABRA, A.; DANTAS, L.E.P.B.T. Space definition for match analysis in soccer. International Journal of Performance Analysis in Sports, Cardiff, v.6, n.2, p.97-113, 2006.

TENGA, A.; HOLME, I.; RONGLAN, L.T.; BAHR, R. Effect of playing tactics on achieving score-box possessions in a random series of team possessions from Norwegian professional soccer matches. Journal of Sports Sciences, London, v.28, n.3, p.245-55, 2010a.

. Effect of playing tactics on goal scoring in Norwegian professional soccer. Journal of Sports Sciences, London, v.28, n.3, p.237-44, 2010b.

TENGA, A.; KANSTAD, D.; RONGLAN, L.T.; BAHR, R. Developing a new method for team match performance analysis in professional soccer and testing its reliability. International Journal of Performance Analysis in Sports, Cardiff, v.9, p.8-25, 2009. VAN INGEN SCHENAU, G.J.; DE GROOT, G.; SCHEURS, A.W.; MEESTER, H.; DE KONING, J.J. A new skate allowing powerful plantar flexions improves performance. Medicine and Science in Sports and Exercise, Madison, v.28, n.4, p.531-35, 1996. WARD, P.; ECCLES, D.W. A commentary on "team cognition and expert team": emerging insights into performance for exceptional team”. International Journal of Sport and Exercise Psychology, Abingdon, v.4, p.463-83, 2006.

WINTER, F. The triple post offense. Englewood Cliffs: Prentice-Hall,1962.

WLOCH, K.; BENTLEY, P.J. Optimising the performance of a formula one car using a genetic algorithm. Lecture Notes in Computer Science, Berlin, v.3242, p.702-11, 2004.

\section{Agradecimentos}

Estes trabalho foi financiado pela FAPESP (Fundação de Amparo à Pesquisa do Estado de São Paulo, Brasil), Processo: 2008/10810-1. 